\title{
Characterization of white electroluminescent devices fabricated using conjugated polymer blends
}

\author{
Do-Hoon Hwang, ${ }^{\text {a) }}$ Moo-Jin Park, and Suk-Kyung Kim \\ Department of Applied Chemistry, Kumoh National Institute of Technology, Kumi 730-701, Korea \\ Nam-Heon Lee \\ Department of Physics, Inha University, Incheon 402-751, Korea \\ Changhee Lee \\ School of Electrical Engineering and Computer Science, Seoul National University, \\ Seoul 151-744, Korea \\ Yong-Bae Kim \\ Liquid Crystal Research Center, Department of Chemistry, Kon-Kuk University, Seoul 143-701, Korea \\ Hong-Ku Shim \\ Center for Advanced Functional Polymers, Department of Chemistry and School of Molecular \\ Science (BK21), Korea Advanced Institute of Science and Technology, Taejon 305-701, Korea
}

(Received 4 November 2003; accepted 2 April 2004)

\begin{abstract}
We report the characterization of white light emitting devices fabricated using conjugated polymer blends. Blue emissive poly[9,9-bis(4'-n-octyloxyphenyl)fluorene-2, 7-diyl-co-10-(2'-ethylhexyl)phenothiazine-3,7-diyl] [poly(BOPF-co-PTZ)] and red emissive poly(2-(2'-ethylhexyloxy)-5-methoxy-1,4-phenylenevinylene) (MEH-PPV) were used in the blends. The inefficient energy transfer between these blue and red light emitting polymers (previously deduced from the photoluminscence (PL) spectra of the blend films) enables the production of white light emission through control of the blend ratio. The PL and electroluminescence (EL) emission spectra of the blend systems were found to vary with the blend ratio. The EL devices were fabricated in the indium tin oxide [poly(3,4-ethylenedioxy-thiophene)-poly(styrenesulfonate)] (ITO/PEDOT-PSS)blend/LiF/Al configuration, and white light emission was obtained for one of the tested blend ratios.
\end{abstract}

\section{INTRODUCTION}

Conjugated polymers have attracted much research interest in science and technology in the past few decades as electro-active materials for diverse applications that include transistors, ${ }^{1-3}$ photovoltaic devices, ${ }^{4}$ nonlinear optical devices, ${ }^{5}$ and polymer light-emitting diodes (PLEDs) ${ }^{6-10}$ In particular, their use in electroluminescence applications has been extensively studied to realize thin, efficient, and stable displays with a wide viewing angle and fast response. Organic electroluminescence (EL) applications such as automotive, mobile, and television displays continue to make progress toward commercial production. Poly(fluorene)s (PFs) ${ }^{1-15}$ and poly(1,4-phenylenevinylene)s (PPVs ${ }^{16-20}$ are promising candidates for PLEDs. PPV derivatives are normally green and red emitting materials; the introduction of electron-donating or electron-withdrawing groups into these derivatives can be used to vary their emitting color.

\footnotetext{
a) Address all correspondence to this author.

e-mail: dhhwang@kumoh.ac.kr

DOI: $10.1557 / J M R .2004 .0261$
}

Although PFs are blue emitting materials, color tuning of these materials is easily obtained by copolymerization with low band gap comonomers. ${ }^{15}$

White light emitting diodes have attracted significant research interest due to their applications in full color displays combined with a color filter, in backlights for liquid crystal displays, and in meeting various other lighting requirements. White light emission requires the mixing of two complementary colors or three primary colors. Various methods and challenges for generating white light have been reported. The doping method has been widely used to obtain white light. In small molecule devices, a red light emitting material is co-deposited with blue and/or green light emitting materials. ${ }^{21-23}$ Kido et al. have reported that solution-processed polymer devices using composites of blue (B), green $(\mathrm{G})$, and red (R) emitting dyes and poly(vinylcarbazole) emit white light. ${ }^{24}$ In both vacuum-deposited small molecule devices and solution-processed polymer devices, control of the energy transfer between the red, green, and blue dyes is essential and usually requires the introduction of very low levels of doping controls. 
Recently, we found that there is inefficient energy transfer between PF and PPV derivatives. ${ }^{25}$ Therefore, it is possible to obtain white light emission from a blend of PF and PPV derivatives by controlling the blend ratio. In this study, we fabricated white light emitting diodes using polymer blends composed of two emission components. A highly efficient blue light emitting PF copolymer, poly[9,9-bis(4'-n-octyloxyphenyl)fluorene-2,7diyl-co10-(2-ethylhexyl)phenothiazine-3,7-diyl] [poly(BOPF-co-PTZ)], and a well known red light emitting PPV derivative, poly[2-(2'-ethylhexyloxy)-5-methoxy1,4-phenylenevinylene] (MEH-PPV), were used in the blends. The synthetic routes of the polymers used in the blend system are shown in Fig. 1.

\section{EXPERIMENTAL}

\section{A. Synthesis of poly(84BOPF-co-16PTZ)}

Poly(84BOPF-co-16PTZ) was synthesized using nickel (0) mediated polymerization. The feed ratio of phenothiazine monomer was $10 \mathrm{~mol} \%$ of the total amount of monomer, and the total amount of reactant was $1.8 \mathrm{mmol}$. Each Schlenk tube containing $5 \mathrm{~mL}$ of dimethylformimide (DMF), bis(1,5-cyclooctadienyl) nickel(0), 2,2'-dipyridyl, and 1,5-cyclooctadiene (the last three in a molar ratio of $1: 1: 1)$ was kept under argon at $80{ }^{\circ} \mathrm{C}$ for $30 \mathrm{~min}$. Five milliliters of anhydrous toluene was then added to the mixture. The polymerization was maintained at $80{ }^{\circ} \mathrm{C}$ for $72 \mathrm{~h}$, and then $0.1 \mathrm{~g}$ of 9-bromoanthracene was dissolved in toluene and added to the reaction mixture for end-capping. When the reaction had
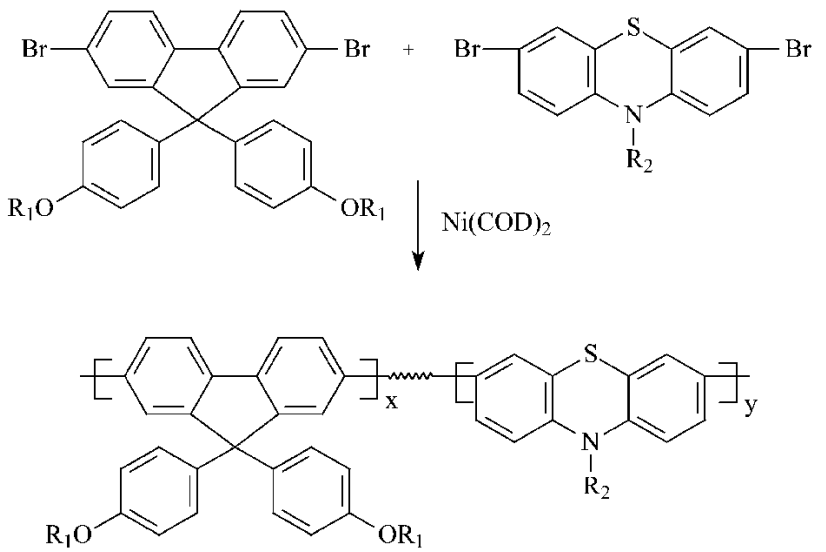

(a) Poly(BOPF-co-PTZ) ( $\mathbf{R}_{1}=$ n-octyl, $R_{2}=$ 2-ethylhexyl)

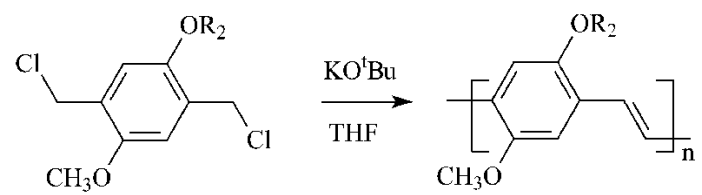

(b)

MEH-PPV ( $\mathbf{R}_{\mathbf{2}}=$ 2-ethylhexyl)

FIG. 1. Synthetic schemes for the (a) poly(BOPF-co-PTZ) and (b) MEH-PPV. finished, the polymer was precipitated from an equivolume mixture of concentrated $\mathrm{HCl}$, methanol, and acetone. The isolated polymer was dissolved in chloroform and precipitated in methanol. The resulting polymer was dissolved in toluene and purified using silica gel column chromatography with toluene as the eluent. Finally, the polymer was purified by Soxhlet extraction with methanol and dried in vacuum. The polymer yield was 59\% after purification. The actual composition of the copolymer was determined by elemental analysis; the proportion of phenothiazine in the copolymer was found to be $16 \%$, so this copolymer is poly(84BOPF-co-16PTZ).

\section{B. Synthesis of MEH-PPV}

A solution of 2,5-bis(chloromethyl)-4-(2-ethylhexyloxy)benzene $(1.0 \mathrm{~g}, 3 \mathrm{mmol})$ in dry tetrahydrofuran (THF) was degassed at room temperature. To the stirred solution a degassed solution of $95 \%$ potassium tertbutoxide $(2.12 \mathrm{~g}, 19 \mathrm{mmol})$ in dry THF $(80 \mathrm{~mL})$ was added dropwise during $20 \mathrm{~min}$ at $20{ }^{\circ} \mathrm{C}$. The reaction mixture became red and viscous during the addition. The highly viscous reaction mixture was stirred at $20{ }^{\circ} \mathrm{C}$ under an atmosphere of argon for $16 \mathrm{~h}$, after which it was evaporated to $2 / 3$ of its initial volume and poured into methanol $(50 \mathrm{~mL})$ with stirring. The resulting red precipitate was filtered, washed with distilled water, dissolved in the minimum quantity of THF $(\sim 100 \mathrm{~mL})$, and then reprecipitated by pouring into methanol $(500 \mathrm{~mL})$; the precipitate was then dried in vacuo at $20{ }^{\circ} \mathrm{C}$ to afford MEH-PPV. The polymer yield was $60 \%$ after purification.

\section{Fabrication and characterization of the EL devices}

The absorption spectra were measured using a Hitachi spectrophotometer model U-3501, and the steady-state photoluminescence spectra were recorded on a Spex FL3-11. The ionization potentials of the polymer films were measured with a low-energy photo-electron spectroscope (Riken-Keiki AC-2). The polymer films were prepared by spin-casting blend solutions containing $1 \%$ of the polymers by weight in chlorobenzene. Uniform and pinhole-free films with a thickness around $80 \mathrm{~nm}$ were easily obtained from the polymer solution. For the double layer device, a modified water dispersion of PEDOT [poly(3,4-ethylenedioxy-thiophene)] doped with poly(styrene sulfonate) (Bayer AG, Leverkusen, Germany) was used as the hole-injection/transport layer. To improve the electron injection, we deposited a thin $\mathrm{LiF}$ layer $(\sim 0.5 \mathrm{~nm})$ onto the polymer film and then deposited the aluminum electrode $(\sim 100 \mathrm{~nm})$ using the thermal evaporator at a pressure of $10^{-6}$ Torr. The device performance was studied by measuring its current-voltage-EL (I-V-L) characteristics, EL spectra, and Commission Internationale d'Eclairage (CIE) coordinates. The I-V-L 
characteristics were measured with a Keithley 236 (Keithley Instruments, Inc., Cleveland, Ohio) sourcemeasure unit and a Keithley 2000 multimeter equipped with a photomultiplier tube (PMT) through an ARC 275 (Acton Research Corp, Acton, Massachusetts) monochromator. The external quantum efficiency of the EL, defined as the ratio of the emitted photons to the injected charges, was calculated from the EL intensity measured with the calibrated $\mathrm{Si}$ photodiode. All processes and measurements described above were carried out in air at room temperature.

\section{RESULTS AND DISCUSSION}

Figure 2 shows the ultraviolet (UV)-visible absorption spectrum of each polymer used in the polymer blends. Poly(84BOPF-co-16PTZ) and MEH-PPV exhibit absorption maxima at 371 and $512 \mathrm{~nm}$, respectively. Optical band gaps were obtained from the absorption edges of each polymer. The blue light emitting poly(84BOPF-co16PTZ) has a wider band gap (2.82 eV) than MEH-PPV $(2.10 \mathrm{eV})$. To determine the highest occupied molecular orbital (HOMO) levels of the polymers, their ionization potentials were measured using photoemission spectroscopy. The measured ionization potentials of poly(84BOPF-co-PTZ) and MEH-PPV were 5.40 and $4.90 \mathrm{eV}$, respectively. The lowest unoccupied molecular orbital (LUMO) levels of the poly(84BOPF-co-16PTZ) and MEH-PPV thin films were estimated to be 2.58 and $2.80 \mathrm{eV}$, respectively. Poly(84BOPF-co-16PTZ) and MEH-PPV thin films exhibit photoluminescence (PL) maxima at $482 \mathrm{~nm}$ (greenish blue) and $575 \mathrm{~nm}$ (orange red), respectively, as shown in Fig. 2.

Several polymer blend systems with different blend ratios were prepared from these two polymers. We prepared several blend systems containing 0.5 to $3.0 \%$ red emitting MEH-PPV in the blue emitting PF copolymer

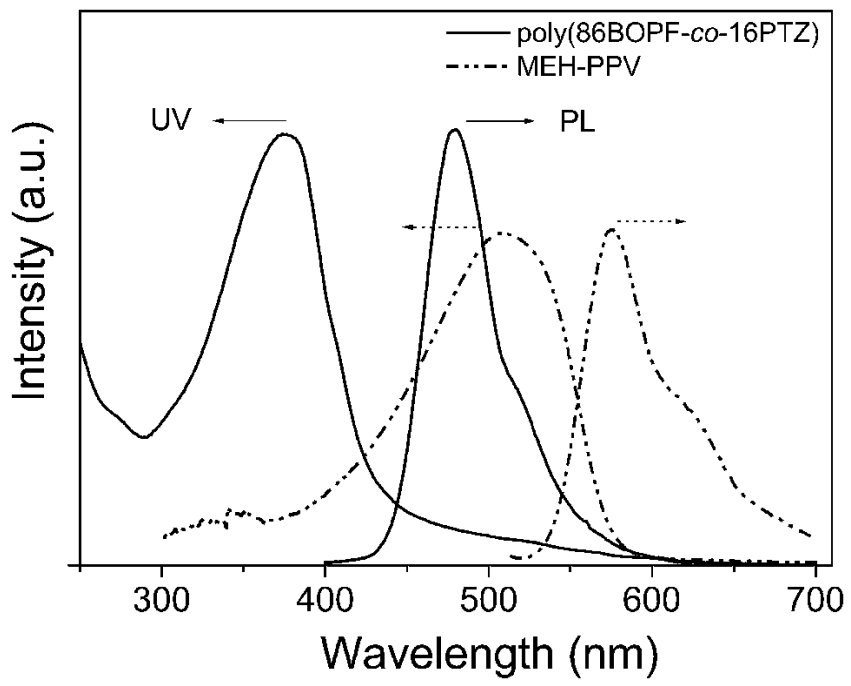

FIG. 2. UV-visible absorption and PL emission spectra of the individual polymer films.

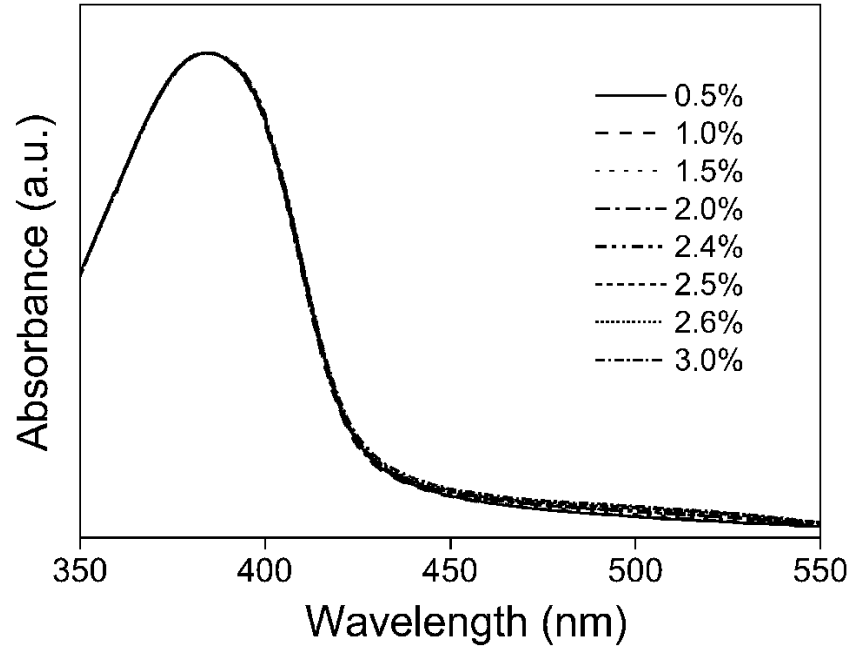

FIG. 3. UV-visible absorption spectra of the thin films of the polymer blends with different blend ratios.

host. Figure 3 shows the UV-visible absorption spectra of the blend systems. Their absorption spectra are almost the same as that of the host PF derivative; only weak absorption due to MEH-PPV was observed, suggesting that most of the absorption was due to the PF copolymer.

Figure 4 shows the PL spectra of the polymer blends. As the ratio of MEH-PPV increases, the intensity of the emission band near $560 \mathrm{~nm}$ increases. In the polymer blend containing 2.5\% MEH-PPV, the intensities of the two emission bands at 490 and $560 \mathrm{~nm}$ are comparable. Considering that the spectral overlaps of poly(BOPF-coPTZ) and MEH-PPV are large enough to produce Förster energy transfer between the polymers, this inefficient energy transfer is likely to be due to a phase separation of the polymer blends, which should be investigated further. In fact, this inefficient energy transfer enables the production of white light emission through control of the blend ratio. Properties of the poly(84BOPF-co-16PTZ) and MEH-PPV are summarized in Table 1. EL devices were fabricated in the (ITO/PEDOT-PSS)blend/LiF/Al configuration. Figure 5 shows the EL spectra of the EL devices fabricated using the polymer blend systems. As in the PL spectra, as the MEH-PPV ratio in the blend system increases the intensity of the emission band at $560 \mathrm{~nm}$ increases. The two emission bands in the EL spectra of the EL device using a polymer blend containing $2.5 \% \mathrm{MEH}-\mathrm{PPV}$ are of comparable intensity, and this device exhibits an efficient white light emission.

One of the major problems of white EL devices is that the emission color varies with the voltage or current that is applied. This variation of the emission color lessens the color purity of such EL devices. To investigate the devices' stability of emission, we measured the voltage dependence of the EL spectra of the devices using 2.5\% blend system. As shown in Fig. 6, the devices exhibit EL 


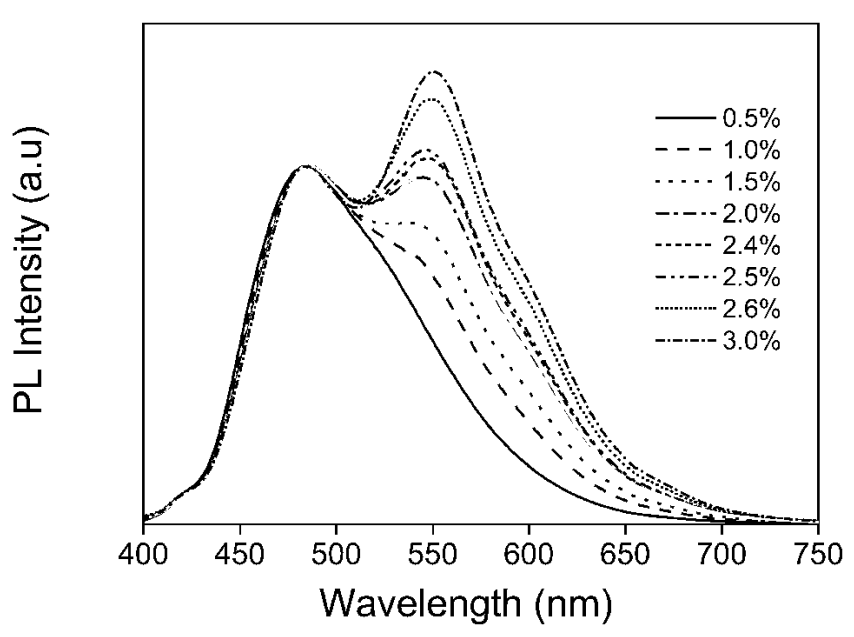

FIG. 4. PL emission spectra of the thin films of the polymer blends with different blend ratios.

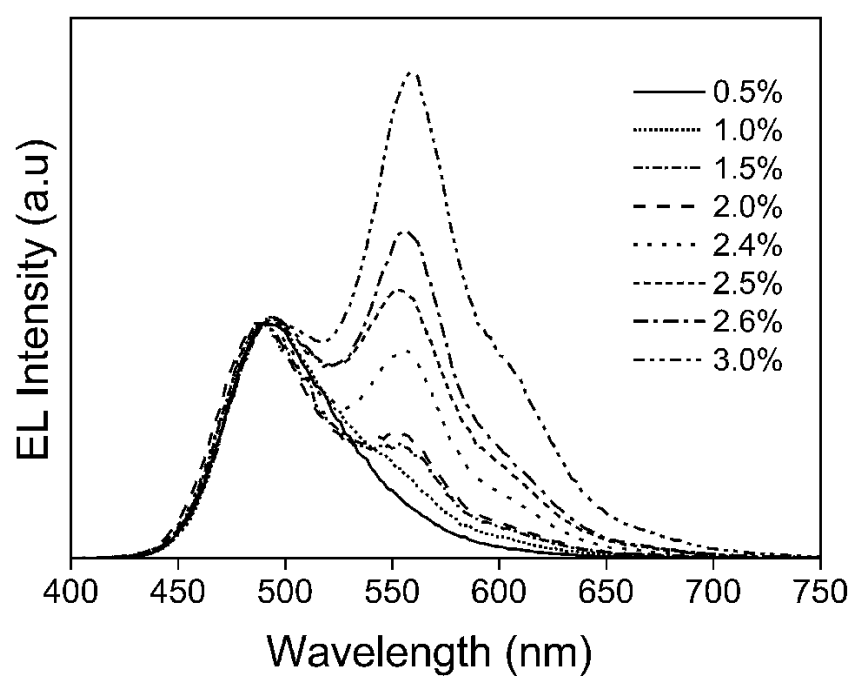

FIG. 5. EL spectra of the devices with ITO/PEDOT/blend/LiF/Al configurations using polymer blends with different blend ratios.

spectra that are stable to variation of the applied current. Among all the EL devices tested in this experiment, the device using the $2.5 \%$ MEH-PPV blend system exhibited the CIE coordinates $(0.32,0.49)$ closest to the standard CIE coordinates for white light emission $(0.33,0.33)$.

Figure 7 shows the current-voltage and luminancevoltage characteristics of the EL devices. The forward current was found to increase with increasing forward bias voltage, and the curve has the same characteristics as those of a typical diode. There was little difference between the I-V-L characteristics of the devices, and light emission from the devices was observed at an applied voltage of less than $10 \mathrm{~V}$. The maximum brightness of the devices ranged from 1580 to $2640 \mathrm{~cd} / \mathrm{m}^{2}$ with external quantum efficiencies of 0.3 to $0.40 \%$. The CIE coordinates measured at $100 \mathrm{~cd} / \mathrm{m}^{2}$ brightness and other device characteristics are summarized in Table II.

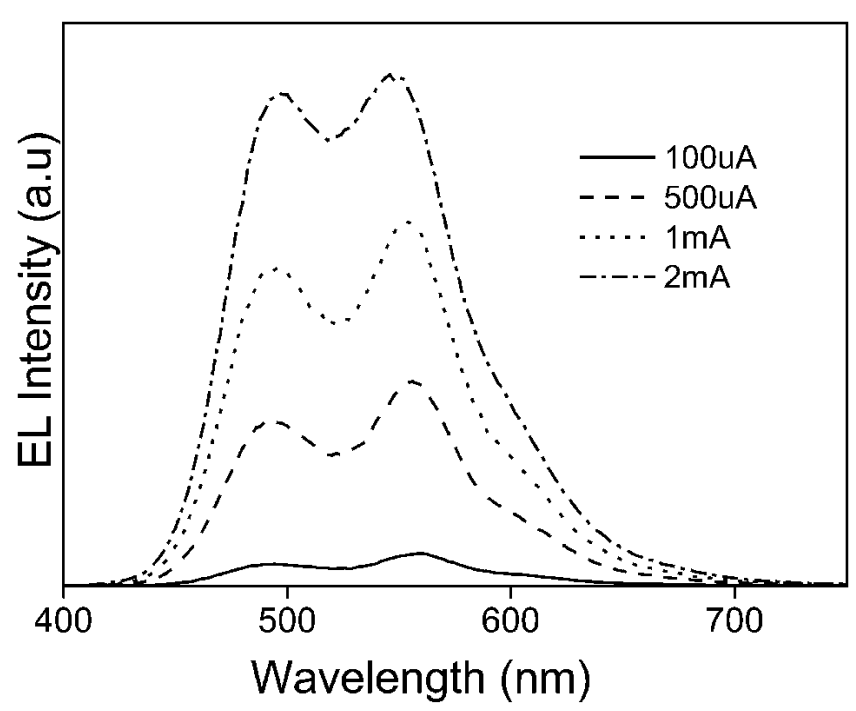

FIG. 6. Current versus EL spectra of the devices using 2.5\% MEH-PPV.
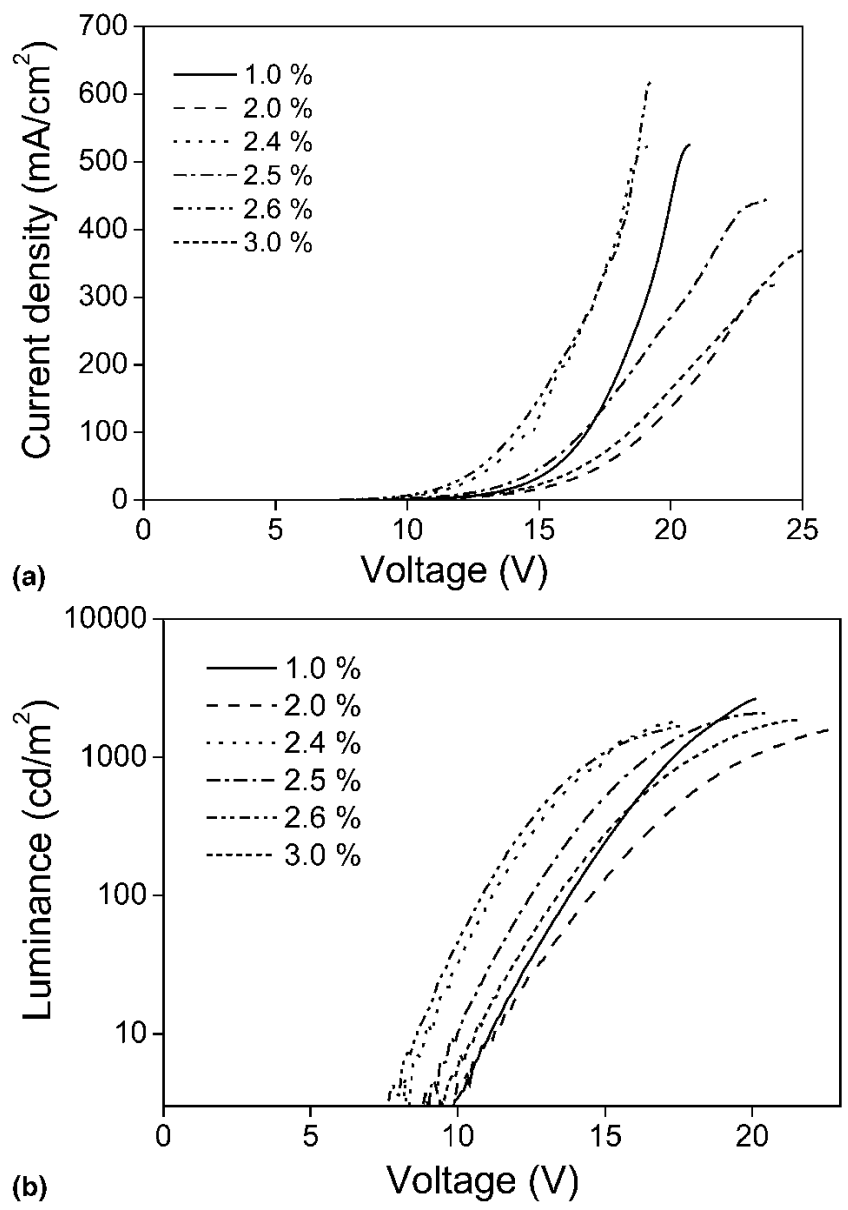

FIG. 7. (a) I-V and (b) L-V curves of the EL devices with ITO/PEDOT/ blend/LiF/Al configurations.

The CIE coordinates of the EL devices using the polymer blends can be further tuned by changing the blend ratio. Our results for the EL devices using the above polymer blends are preliminary, so optimizations of 
TABLE I. Summary of the properties of the polymers used in the blends.

\begin{tabular}{|c|c|c|}
\hline Polymer & Poly(84BOPF-co-16PTZ) & MEH-PPV \\
\hline Average $M_{w}$ & 31,500 & 147,000 \\
\hline PDI & 1.7 & 4.8 \\
\hline $\mathrm{UV} \max (\mathrm{nm})^{\mathrm{a}}$ & 371 & 512 \\
\hline $\mathrm{PL} \max (\mathrm{nm})^{\mathrm{a}}$ & 482 & 575 \\
\hline Optical band gap (eV) & 2.82 & 2.10 \\
\hline HOMO level $(\mathrm{eV})^{\mathrm{b}}$ & 5.40 & 4.90 \\
\hline LUMO level $(\mathrm{eV})$ & 2.58 & 2.80 \\
\hline
\end{tabular}

${ }^{\mathrm{a}}$ Measured for thin films on fused quartz plates.

${ }^{\mathrm{b}}$ Determined by photoemission spectroscopy using Riken Keiki AC-2. PDI, polydispersity index; PL, photoluminescence; HOMO, highest occupied molecular orbital; LUMO, lowest unoccupied molecular orbitals.

TABLE II. Summary of the device characteristcs.

\begin{tabular}{lccccc}
\hline \hline & & & & Power & CIE \\
Polymers $^{\mathrm{a}}$ & $\begin{array}{c}\mathrm{V}_{\text {turn-on }} \\
(\mathrm{V})\end{array}$ & $\begin{array}{c}\text { Luminance } \\
\left(\mathrm{cd} / \mathrm{m}^{2}\right)\end{array}$ & $\begin{array}{c}\text { Efficiency } \\
(\%)\end{array}$ & $\begin{array}{c}\text { efficiency } \\
(\mathrm{lm} / \mathrm{W})\end{array}$ & $\begin{array}{c}\text { coordinates } \\
(\mathrm{x}, \mathrm{y})\end{array}$ \\
\hline Blend 1.0\% & 9.9 & 2640 & 0.31 & 0.15 & $(0.19,0.45)$ \\
Blend 2.0\% & 9.9 & 1580 & 0.34 & 0.16 & $(0.23,0.42)$ \\
Blend 2.4\% & 8.2 & 1800 & 0.31 & 0.22 & $(0.28,0.46)$ \\
Blend 2.5\% & 9.0 & 2080 & 0.39 & 0.24 & $(0.32,0.49)$ \\
Blend 2.6\% & 7.7 & 1670 & 0.30 & 0.22 & $(0.32,0.51)$ \\
Blend 3.0\% & 9.4 & 1860 & 0.40 & 0.25 & $(0.36,0.51)$ \\
\hline \hline
\end{tabular}

${ }^{a}$ The percentage indicates the wt $\%$ of MEH-PPV in the blend system.

${ }^{\mathrm{b}}$ The CIE coordinates were measured at $100 \mathrm{~cd} / \mathrm{m}^{2}$ brightness.

the device and the blend ratio are needed to obtain high efficiency white light emission, which are under investigation.

\section{CONCLUSIONS}

Blends of light emitting polymers were prepared to produce white light emission. EL devices using polymer blends of poly(84BOPF-co-16PTZ) and MEH-PPV were found to exhibit current- and voltage-independent stable white light emission. A polymer blend contained 2.5\% MEH-PPV was found to exhibit an EL spectrum that is closer to the standard white than those of the devices using other blend ratios. A maximum brightness of $2080 \mathrm{~cd} / \mathrm{m}^{2}$, an external quantum efficiency of $0.39 \%$, and CIE coordinates of $(0.32,0.49)$ at $100 \mathrm{~cd} / \mathrm{m}^{2}$ were obtained with the device. We suggest that inefficient energy transfer between blue light emitting PF derivatives and low band gap PPV derivatives can be taken advantage of to produce efficient white light emitting diodes.

\section{ACKNOWLEDGMENT}

We gratefully acknowledge funding from the Advanced Backbone IT technology development project supported by the Ministry of Information \& Communication of the Republic of Korea.

\section{REFERENCES}

1. R. Wisnieff: Display technology: Printing screens. Nature 394, 225 (1998).

2. H. Shirringhaus, N. Tessler, and R.H. Friend: Integrated optoelectronic devices based on conjugated polymers. Science 280, 1741 (1998).

3. H.E. Katz, Z. Bao, and S.L. Gilat: Synthetic chemistry for ultrapure, processable, and high-mobility organic transistor semiconductors. Acc. Chem. Res. 34, 359 (2001).

4. J.J. Halls, C.A. Walsh, N.C. Greenham, E.A. Marseglia, R.H. Friend, S.C. Moratti, and A.B. Holmes: Efficient photodiodes from interpenetrating polymer networks. Nature 376, 498 (1995).

5. P.N. Prasad and D.J. Williams: Introduction to Nonlinear Effects in Monomers and Polymers, (John Wiley \& Sons, New York, 1991).

6. J.H. Burroughes, D.D.C. Bradley, A.R. Brown, R.N. Marks, K. Mackay, R.H. Friend, P.L. Burn, and A.B. Holmes: Lightemitting diodes based on conjugated polymers. Nature 347, 539 (1990).

7. G. Gustafsson, Y. Cao, G.M. Treacy, F. Klavetter, N. Colaneri, and A.J. Heeger: Flexible light-emitting diodes made from soluble conducting polymers. Nature 357, 477 (1992).

8. P.L. Burn, A.B. Holmes, A. Kraft, D.D.C. Bradley, A.R. Brown, R.H. Friend, and R.W. Gymer: Chemical tuning of electroluminescent copolymers to improve emission efficiencies and allow patterning. Nature 356, 47 (1992).

9. A. Kraft, A.C. Grimsdale, and A.B. Holmes: Electroluminescent conjugated polymers-seeing polymers in a new light Angew. Chem. Int. Ed. 37, 402 (1998).

10. U. Mitschke and P. Bäuerle: The electroluminescence of organic materials. J. Mater. Chem. 10, 1471 (2000).

11. D. Neher: Polyfluorene Homopolymers: Conjugated liquidcrystalline polymers for bright blue emission and polarized electroluminescence. Macromol. Rapid. Commun. 22, 1365 (2001).

12. U. Scherf and E.J.W. List: Semiconducting polyfluorenes towards reliable structure-property relationships. Adv. Mater. 14, 477 (2002).

13. A. Pogantsch, F.P. Wenzl, E.J.W. List, G. Leizing, A.C. Grimsdale, and K. Müllen: Polyfluorenes with dendron side chains as the active materials for polymer light-emitting devices. Adv. Mater. 14, 1061 (2002).

14. N.S. Cho, D.H. Hwang, J.I. Lee, B.J. Jung, and H.K. Shim: Synthesis and color tuning of new fluorene-based copolymers. Macromolecules 35, 1224 (2002).

15. J.H. Lee and D.H. Hwang: Alkoxyphenyl substituted polyfluorene; a stable blue light emitting polymer with good solution processibility. Chem. Commun. 00, 2836 (2003).

16. D.H. Hwang, S.T. Kim, H.K. Shim, A.B. Holmes, S.C. Moratti, and R.H. Friend: Green light-emitting diode from poly(2dimethyloctylsilyl-1,4-phenylenevinylene). J. Chem. Soc. Chem. Commun. 00, 2241 (1996).

17. H. Becker, H. Spreitzer, K. Ibrom, and W. Kreuder: New insights into the microstructure of GILCH-polymerized PPVs. Macromolecules. 32, 4925 (1999).

18. H. Becker, H. Spreitzer, W. Kreuder, E. Kluge, H. Schenk, I. Parker, and Y. Cao: Soluble PPVs with enhanced performance a mechanistic approach. Adv. Mater. 12, 43 (2000).

19. D.H. Hwang and H.K. Shim: Luminescence properties of a liquid crystalline poly[2,5-bis(dimethyloctylsilyl)-1,4-phenylenevinylene]. Thin Solid Film 417, 166 (2002).

20. D.H. Hwang, J.D. Lee, J.M. Kang, S. Lee, C.H. Lee, and S.H. Jin: Synthesis and light-emitting properties of poly $(9,9$-di-n-octylfluorenyl- 2,7-vinylene) and PPV copolymers. J. Mater. Chem. 13, 1540 (2003). 
21. A. Dodabalapur, L.J. Rothberg, and T.M. Miller: Color variation with electroluminescent organic semiconductors in multimode resonant cavities. Appl. Phys. Lett. 65, 2308 (1994).

22. J. Kido, M. Kimura, and K. Nagai: Multilayer white light-emitting organic electroluminescent device. Science 267, 1332 (1995).

23. R.S. Deshpande, V. Bulovic, and S.R. Forrest: White-light-emitting organic electroluminescent devices based on interlayer sequential energy transfer. Appl. Phys. Lett. 75, 888 (1999).
24. J. Kido, H. Shionya, and K. Nagai: Single-layer white lightemitting organic electroluminescent devices based on dyedispersed poly(N-vinylcarbazole). Appl. Phys. Lett. 67, 2281 (1995).

25. D.H. Hwang, J.H. Lee, J.I. Lee, C.H. Lee, and Y.B. Kim: White electroluminescent devices using polymer blends. Mol. Cryst. Liq. Cryst. 405, 127 (2003). 